\title{
Characterization of the indoor/outdoor to indoor MIMO radio channel at 2.140 $\mathrm{GHz}^{*}$
}

\author{
Hung Tuan Nguyen, Jørgen Bach Andersen and Gert Frølund Pedersen \\ Department of Communication Technology, Niels Jernes Vej 12, DK-9220 Aalborg, Denmark
}

\begin{abstract}
In this paper the radio channel characteristics of the 8x4 MIMO system consisting of a base station and a small terminal equipped with multiple antennas for indoor-indoor and outdoor-indoor scenarios are presented. We study the large-scale variation and small-scale characteristics of the measured channel coefficients. Although the mean received power is very much dependent on the measured location, the channel capacity seems to be unchanged when the receiver's location is altered. The data collected from different scenarios (e.g. measurement locations, antenna setting) were used to investigate the advantage of having the knowledge of the channel at both ends of the transmission link. It is shown that using the water filling algorithm there is indeed an increase in the channel capacity. At low SNR, the benefit of knowing the channel at both link ends observed in the measurement data is much higher than which can be obtained in the channel matrix with usual assumption on identical independently distributed components. Using the small-scale and large-scale information in the formulation of the channel capacity we show that in our measurement, the variation of the mean received power has a greater influence on the change of the overall system performance than the change in the environmental multipath scattering property.
\end{abstract}

Keywords: MIMO, measurements, antenna configuration, mobile terminal antennas

\section{Introduction}

Applying multiple antennas at both the transmit and receive side have emerged as a new approach that promises a huge capacity gain and performance improvement. In these Multiple Input Multiple Output (MIMO) systems, the inherent multipath fading components in wireless communication are processed in a smart way such that the small-scale fading is mitigated and parallel channels created. Because of its potential, enormous amount of work has been carried out in order to understand the behavior of MIMO channel propagation. Channel measurements become very crucial for such understanding as well as for the derivation of models that can be used for system design and simulation.

Based on the measurement results, the MIMO channel can be characterized by analyzing the scattering richness or more specifically the direction of the multipath components at both link ends of the propagation channel. In this research approach, emphasis is put onto the investigation of the double directional description of the propagation channel. The antennas therefore, become independent of the analyzed results. The Time Delay of Arrival (TDoA), the Angle of Arrival (DoA), the Angle of Departure (DoD) and the Angle Power Spectrum (APS) are very important metrics in extracting the double directional information of the channel, see for example (Steinbauer, 2002), (Thoma, 2002), (Medbo, 2001) and (Fugen, 2002).

On the other hand, studies on the characteristics of the MIMO systems with the transmitting and receiving antennas taken as integral parts of the radio channel also gains a lot of interest. The results obtained from this research direction are very useful for evaluating the performance of the MIMO system as a whole. Most of the existing research is focused on the small-scale variation (multipath richness) of the MIMO radio channel in terms of the channel correlation coefficients and/or the channel capacity. There are a number of indoor to indoor or outdoor to outdoor channel characteristics results reported in the literature such as (Kermoal, 2000), (Yu, 2001), (Kivinen, 2002), (Kyritsi1, 2002) and (Chizhik, 2003). Only recently, McNamara et.al. (McNamara, 2002), Wallace et.al. (Wallace, 2002) and Svantesson et.al (Svantesson, 2003) have investigated the influence of the large-scale variation (mean received power) on the performance of the MIMO system. However, there is a lack of measurement results for outdoor to indoor, the scenario in which it is expected to have most applications in 3G systems. Moreover, not many measurement results of real MIMO systems have been published.

\footnotetext{
* This work is partly supported by Aalborg University under the Phd Fellowship grant.
} 
Knowledge on the influences of the practical constraint to the performance of the real MIMO systems is therefore limited.

One of the main objectives of this paper is to assess the performance of a real MIMO system consisting of a base station (BS) and small receiving terminal operating in some real life measurement scenarios. Based on the measurements of indoor to indoor and outdoor to indoor of an 8x4 MIMO system we investigate the multipath richness of the radio channel and the variation of the mean received power. The two parameters are first separately evaluated and later their joint contributions to the overall MIMO performance in terms of the channel capacity are assessed. Throughout the paper, we highlight the importance of including both small-scale variation and large-scale variation in evaluating the performances of MIMO systems. Using the measured data the advantage of having the knowledge of the channel at both ends of the transmission link is discussed. We estimate the improvement or the gain in the channel capacity when the channel is known at the transmitter as compared with the case where it is unknown. A simple formula approximating this gain is also derived.

The rest of the paper is organized in the following way. Firstly, the measurement environment and equipments setup are described in section 2. Having the measured data collected from different scenarios such as receiver's location, receiving antenna setting, we investigate the variation of the mean received power in section 3 . Next, the multipath richness of the measured radio channel is assessed by means of the theoretical channel capacity in section 4 . The advantage of knowing the channel at both ends of the transmission link is also investigated in this section. In section 5, including both small-scale and large-scale information of the measured radio channel in the formulation of the channel capacity we then evaluate the performance of the measured MIMO system. In the last section we wrap up the paper by some conclusions and remarks.

\section{Measurement environment and system set-up}

Center for Personal Kommunication's (CPK) sounder system is built on post-processing and real antenna array technology. A code phase offsetting technique with the use of pseudo noise sequence $(\mathrm{PN})$ is applied. In our measurement campaign a PN sequence of length 511 bits with the chip rate $7.665 \mathrm{MHz}$ was transmitted at a frequency of $2140 \mathrm{MHz}$. It was then sampled at the rate of 15.36 $\mathrm{MHz}$ and the sampled data was stored in a hard disk. There, the data can be further processed in order to get the complex channel impulse responses (IR). The obtained channel data was compensated for all system components. We note that the center frequency and the measurement bandwidth are comparable to the standard center frequency and bandwidth of 3G WCDMA systems.

At the transmit side, we used 8 outputs antenna array, a BS antenna prototype made by ALLGON in Sweden. In this BS antenna both polarization and space diversity are applied. At the receive side, prototypes of the handset and the laptop were deployed. The setup of the handset, laptop and BS's position are illustrated in figure 1. The handset is equipped with 4 patch antennas at the four corners. The distance between the vertical antennas (A1-B1, A2-B2) in the handset is 0.5 wavelength and it is 0.25 wavelength for the horizontal antennas (A1-A2, B1-B2). Meanwhile four patch antennas were arranged as a linear array with an equal distance of 0.5 wavelength. They were mounted at the top edge of the open lid of a prototype laptop. The conducting cable was replaced by optical fibre to avoid radiation disturbance. The handset and laptop were mounted on a sledge capable of moving them linearly in a track over a distance of $1.64 \mathrm{~m}(\approx 11$ wavelengths $)$ with a speed of $23.4 \mathrm{~mm} / \mathrm{s}$. The influence of the user was not considered in the measurement campaign as both the handset and laptop are mounted on a wooden stick in free space. The sampling distance was $7 \mathrm{~mm}$ which made up 20 samples per wavelength. This gives a total number of $32 \times 234$ channel impulse responses at one measured location.

The layout of the measured site was an office building with number of rooms on the same floor. Each room is surrounded by walls made of plasterboard with glass windows. Inside the room, there were several tables chairs and metal furniture (cabinets, measurement equipment, and trolleys) which made a fairly rich scattering environment around the receiver. Three placements of the BS that form an indoor-indoor and two outdoor-indoor measurement scenarios were considered. The total transmitted 
power for the indoor-indoor and outdoor-outdoor scenario were $-16 \mathrm{dBm}$ and $+14 \mathrm{dBm}$ respectively. During the measurements, except the $4^{\text {th }}$ location, the laptop was mounted so that the main beams of the antennas were perpendicular to the corridor's axis. They were faced toward the main entrance door of the room, which was left open during the measurement campaign. The handset antennas' main radiation patterns were always parallel to the corridor's axis (see figure 2).

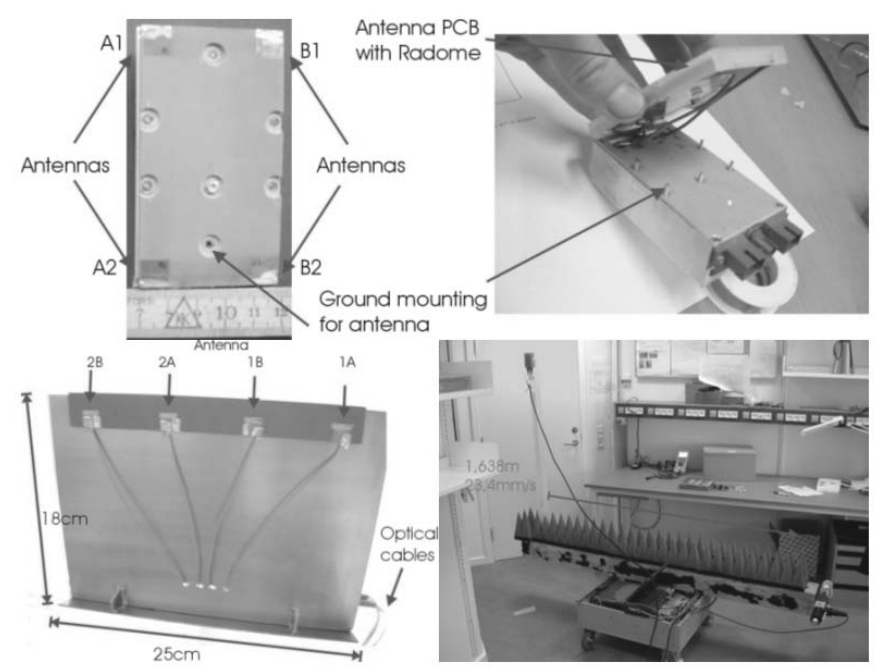

a. Setup of the Handset \& Laptop

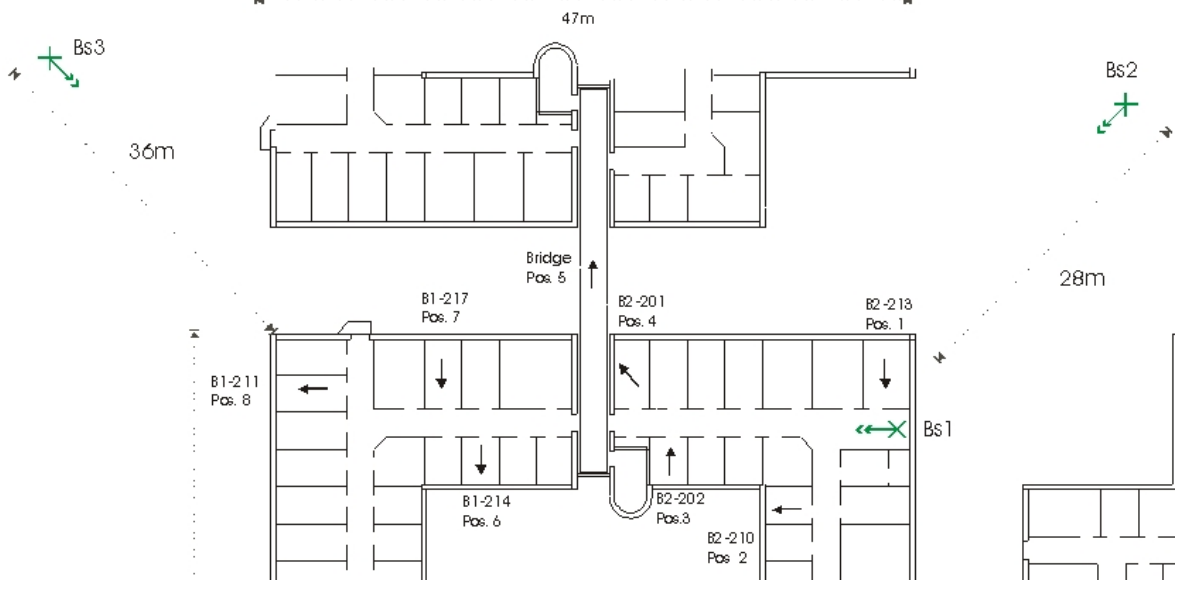

b. Floor plan of the measurement site

Figure 1. Setup and the location of the handset and base station in the measurement

In all measurement locations the distance from the center of the handset and laptop to the floor was $1.69 \mathrm{~m}$. For indoor-indoor measurement the height of the BS was $1.75 \mathrm{~m}$ and it was $5.7 \mathrm{~m}$ for outdoor to indoor measurement.

\section{Large-scale variation or mean received power characteristics}

The mean received power at one measured location is calculated as

$$
\overline{P_{\text {received }}}=\frac{1}{N N_{t} N_{r}} \sum_{n=1}^{N} \sum_{i=1}^{N_{t}} \sum_{j=1}^{N_{r}} \sum_{l=1}^{L}\left|I R_{i j}(l)\right|^{2}
$$

where $L=60$ is the number of taps of the IR, $N_{t}=8$ is the number of transmitting antennas, $N_{r}=4$ is the number of receiving antennas, and $N=234$ is the number of measurement snapshots 


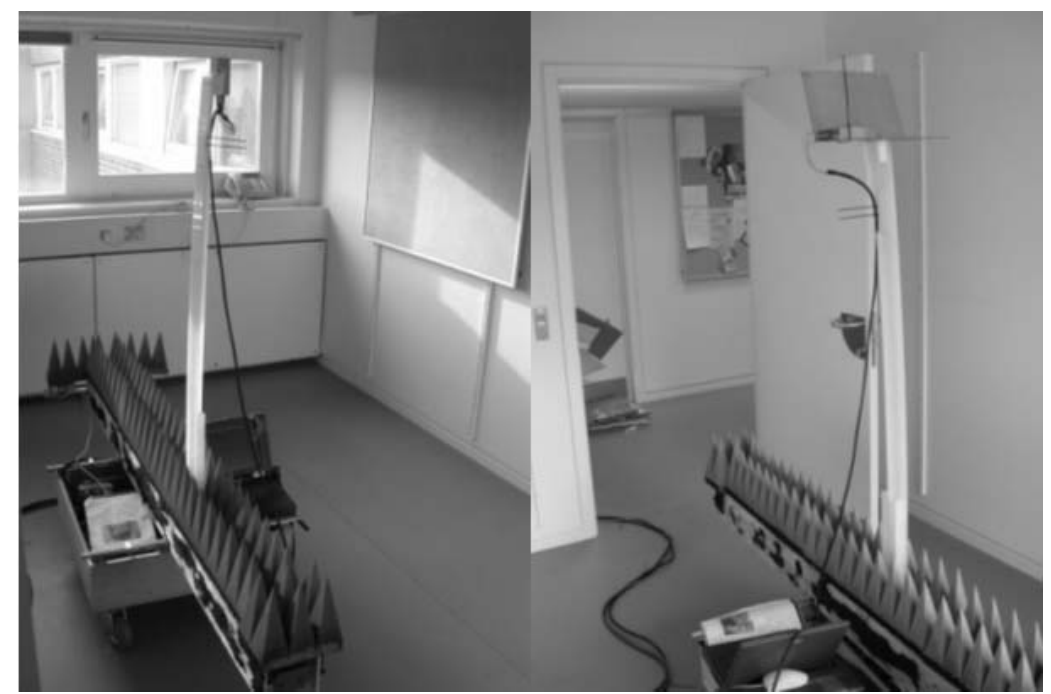

Figure 2. Orientation of the handset and laptop in the measurement campaign

at one location, $I R_{i j}$ is the measured channel impulse response between $i^{\text {th }}$ transmitting antenna and $j^{\text {th }}$ receiving antenna. The mean received power at each measured location for both the handset and laptop are illustrated in figure 3. Note that this is also the mean received power of one SISO link.

The mean received power is obviously site dependent. For indoor-indoor measurements (BS1) the amount of received power tends to decrease along the corridor. At the same measured location, higher mean received power was observed at the laptop rather than at the handset. The difference in the antenna orientations as mentioned in section 2 might be the main source of the $4 \mathrm{~dB}$ increase in the mean received power at the laptop as compared to that at the handset.

For outdoor-indoor environment it has been previously confirmed that the major part of the signal energy comes through the openings in the building such as windows and main door entrance (Knudsen, 2002). Those measured positions where the main beams of the receiving antennas are oriented toward to the openings will receive the most energy. In our outdoor-indoor measurement scenarios (BS2 and BS3), the fact that antennas at the handset have wider radiation patterns (almost omnidirectional) than those in the laptop (more directional) together with their perpendicular orientations could be the reasons for a higher mean received power observed at the handset than that at the laptop (in the order of $5 \mathrm{~dB})$.

\section{Radio multipath richness or the small-scale variation characteristics}

\subsection{Channel Coefficients Characteristics}

To quantify the time dispersion of the channel, we have assessed the root mean square (RMS) delay spread in all measured locations. To avoid the quantization and the correlation noise a threshold of $30 \mathrm{~dB}$ is used for the power delay profile (PDP). We found that for indoor-indoor scenario in all measured locations, around $80 \%$ to $90 \%$ of the RMS delay spread is lower than the excess delay resolution of $0.135 \mu \mathrm{s}$ which is an inversion of the PN chip rate. For the outdoor-indoor case, only $30 \%$ of all the paths have the time dispersion larger than the excess delay resolution. Therefore we decided to use the narrowband information of the channel for the following analysis. The channel coefficient is derived by summing up all $L=60$ taps out of 90 available taps of the measured IR as the remaining taps often contain the quantization and correlation noise other than useful multipath information.

$$
h_{i j}=\frac{1}{L} \sum_{l=1}^{l=L} I R_{i j}(l)
$$




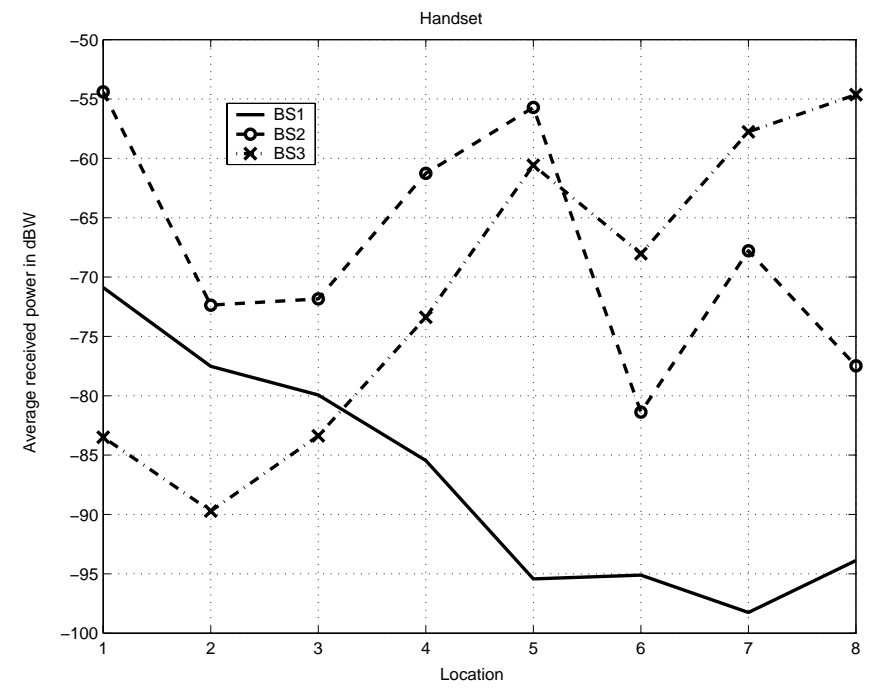

a. Handset

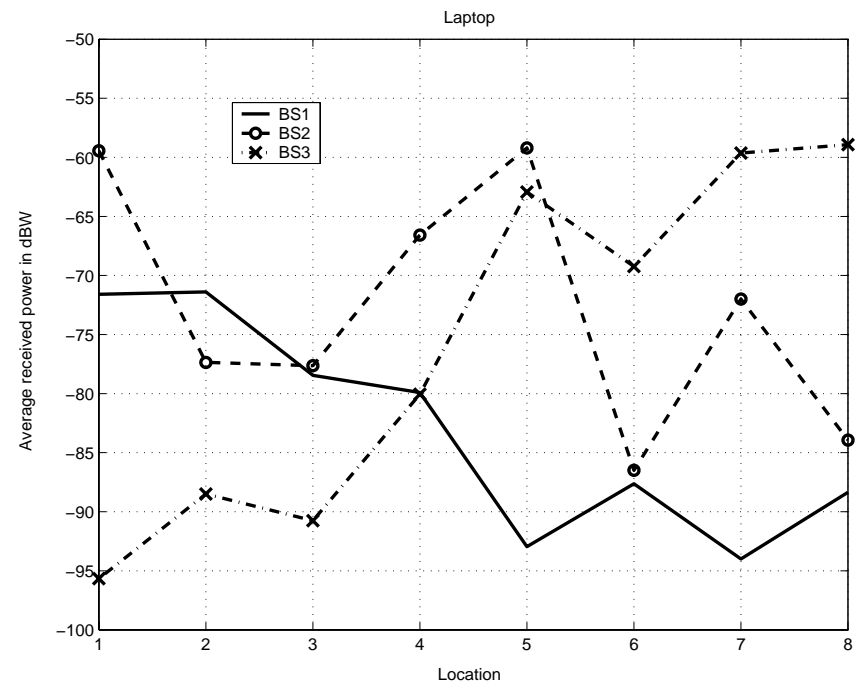

b. Laptop

Figure 3. The mean received power of the handset and laptop at different measured locations

To assess the multipath richness of the measured MIMO radio channel we need to normalize the measured channel matrix. In the normalization process we simply ignore the path loss effect or specifically the amount of received power so that the multipath richness property of the radio channel is highlighted. Herein the channel matrix will be normalized such that

$$
H=\frac{H_{\text {measured }}}{\sqrt{\frac{1}{N_{t} N_{r}} \sum_{i=1}^{N_{t}} \sum_{j=1}^{N_{r}}\left|h_{i j}\right|^{2}}}
$$

This normalization implicitly indicates that the square of the Frobenius norm of the normalized matrix $\mathrm{H}$ will be equal to $N_{t} \times N_{r}$.

Having the channel coefficients it is now possible to study the marginal distribution of their phase and magnitude. From the floor plan of the measurement site (figure 1.b.), it is clear that the channel coefficients must not contain a strong line of sight (LOS) characteristics. We use the data collected from two measurement scenarios namely the indoor-indoor measurement (BS1) with the laptop as the receiver at location 8 and the outdoor-indoor measurement (BS3) with the handset as the receiver at location 1 to study their magnitude and phase distribution. The reason for selecting these two measurement scenarios is that they are, from the ray tracing point of view, most likely having a strong 
LOS property. The results are shown in figure 4. The distribution of the magnitude of the channel coefficients for the indoor-indoor shows a weak LOS characteristics with the best fit Ricean $\mathcal{K}$-factor of 3.5dB. As it can be seen the empirical PDF almost follows the analytical Rayleigh distribution for the outdoor-indoor scenario. The phases of the channel coefficients, although not illustrated here appear to be uniformly distributed over $[0 . .2 \pi]$.

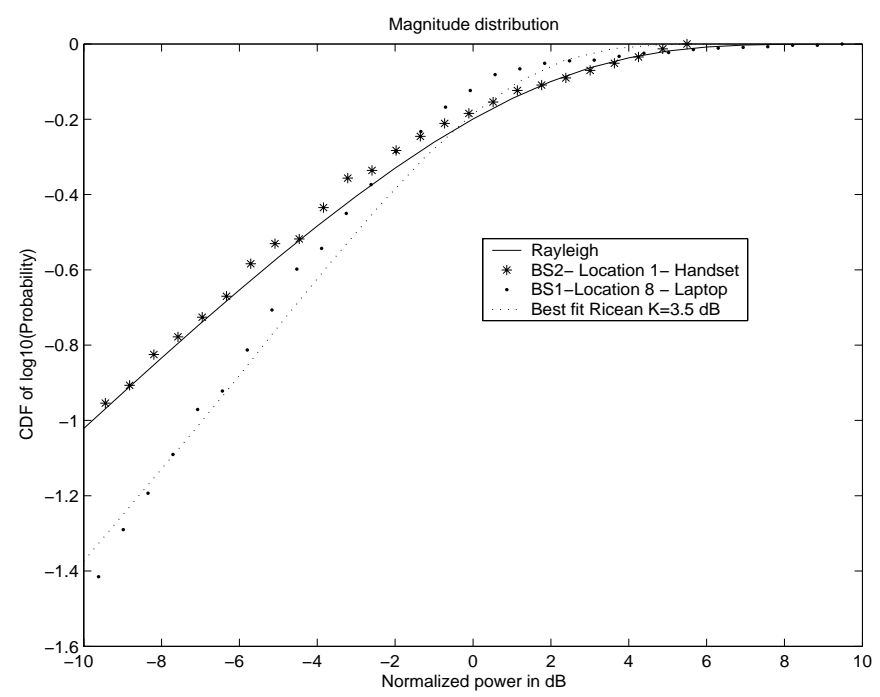

Figure 4. Cumulative distribution function of the magnitude of the $8 \times 4$ MIMO channel coefficients

One motivation for deploying the MIMO technique is to obtain orthogonal subchannels and therefore increasing the channel throughput at the same transmitted power and bandwidth by utilizing the radio multipath richness. Hereafter we use the eigenvalue decomposition technique as a tool to study the distribution of the subchannels' gains as well as the multipath richness property of the measured MIMO channel. In order to gather enough samples for statistical analysis, for each placement of the BS we use the data collected from all measured locations of the receiver. The distributions of the eigenvalues or subchannels' gains are illustrated in figure 5 .

In the same figure the distributions of the eigenvalues in the case where the channel coefficient is identical independently distributed (IID) with zero mean and unit variance are also plotted for reference. It is obvious from the figure that the distribution of the eigenvalues based on the measured data spread over larger range than those of the IID case. The spreads of the eigenvalues calculated as the difference in $\mathrm{dB}$ between the median value of the largest and smallest eigenvalues changes from $9 \mathrm{~dB}$ to $23 \mathrm{~dB}$ for the IID case and the measurement respectively. Interestingly the largest eigenvalue obtained from measurement has higher value than that of the IID case. This situation might be resulted from one of these main reasons:

i) A non line of sight (NLOS) environment with correlated channel that increases the largest eigenvalue.

ii) A environment that has strong LOS property (high $\mathcal{K}$-factor) that reduces the number of non zero eigenvalues.

iii) A environment with correlated channel and a slight LOS characteristics that increases the largest eigenvalue.

In our measurement, the channel coefficients are expected to be partly correlated because of the limit in the separated distance and poor cross polarization discrimination of the transmitting antennas, lack of scattering environment around the transmitter when the BS was outdoor as well as closely placed antennas at the receiver. On the other hand, as has been studied above the measured environment has more NLOS characteristics than LOS. Therefore the third reason seems to be reasonable explanation for the increase in the spread of the measured eigenvalues.

In a MIMO system we would like to have the channels to be decorrelated as much as possible so that full diversity gain can be achieved. The decorrelation degree depends on a number of factors such 


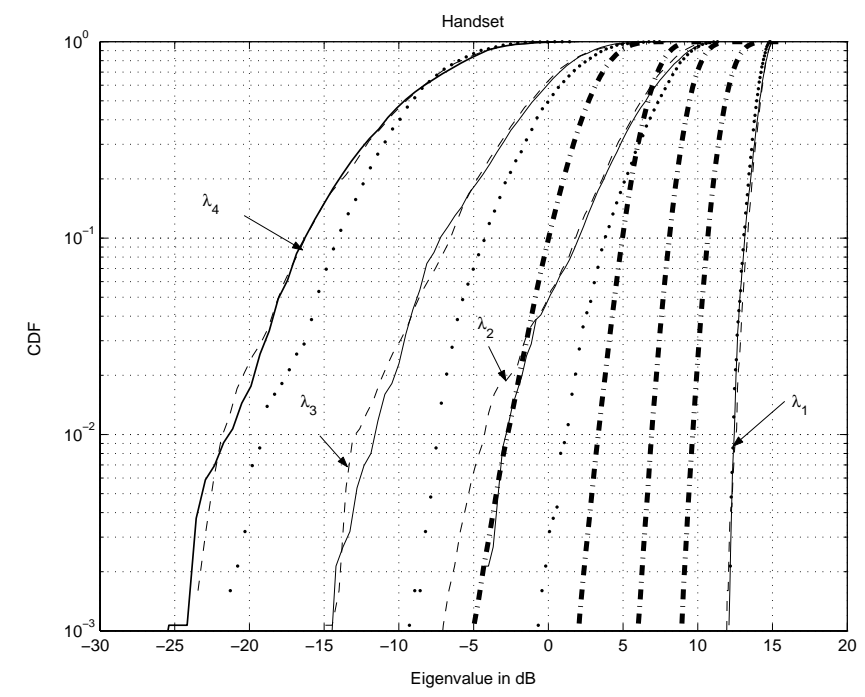

a. Handset

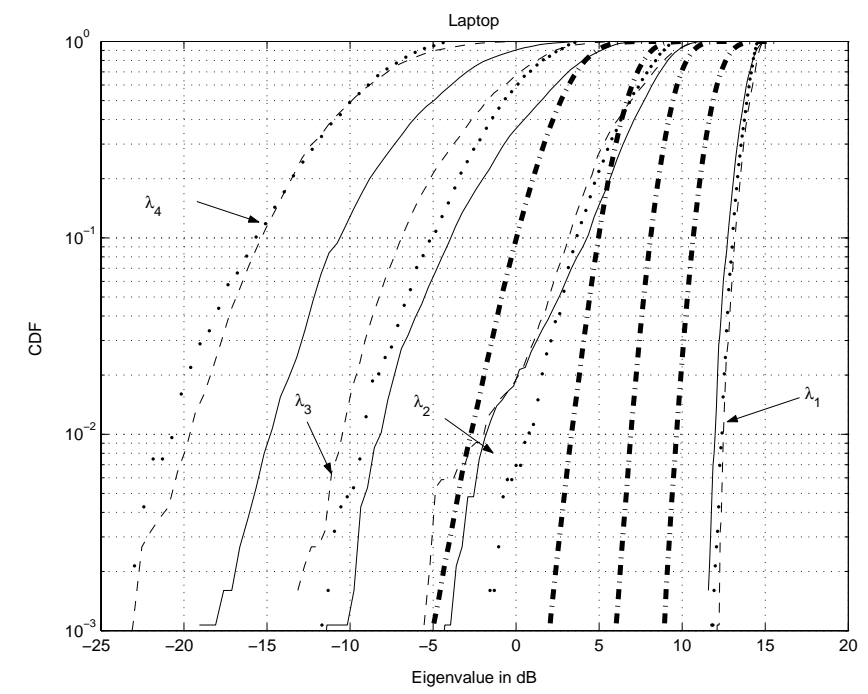

b. Laptop

Figure 5. The distribution of the eigenvalues at three BS's positions, solid lines: BS1, dot lines: BS2, thin dash lines: BS3. For each BS's position the data collected at all receiver's locations are used. Thick dash lines are the distribution of eigenvalues in the IID case

as the multipath environment, the configuration of the transmitting-receiving antennas, interaction of the antenna elements etc. Here we are interested in assessing the decorrealtion of the channels observed from antenna array at the receiver. From these correlation characteristics be it uncorrelated, partly correlated or correlated the benefit of using multiple antennas at a small terminal could be assessed. Based on that it is also possible to compare the correlation of the measured data with the theoretical results. For simplicity we only consider the complex correlation coefficient in the following analysis. The complex correlation coefficient between $j^{\text {th }}$ and $k^{\text {th }}$ receiving antenna is given by

$$
\kappa(j, k)=\frac{1}{N_{t}} \sum_{i=1}^{N_{t}} \frac{E\left(\left(h_{i j}-\overline{h_{i j}}\right)\left(h_{i k}-\overline{h_{i k}}\right)^{*}\right)}{\sqrt{E\left(\left(h_{i j}-\overline{h_{i j}}\right)\left(h_{i j}-\overline{h_{i j}}\right)^{*}\right)} \sqrt{E\left(\left(h_{i k}-\overline{h_{i k}}\right)\left(h_{i k}-\overline{h_{i k}}\right)^{*}\right)}}
$$

where $E($.$) is the expectation operator, (.)* denotes complex conjugate and \overline{(.)}$ denotes the mean value.

In order to gather enough spatial statistic, for each measured location we used all the $32 \times 234$ IRs to calculate receiving spatial correlation coefficient. The variations of the magnitude of the complex 
spatial correlation coefficient at the receivers along the corridor for different placement of the BS are illustrated in figure 6 . Note that the order of the receiver's location has been reversed in the third row so that the $\mathrm{x}$ axis reflects the relative distance from the BS to the handset or to the laptop. It

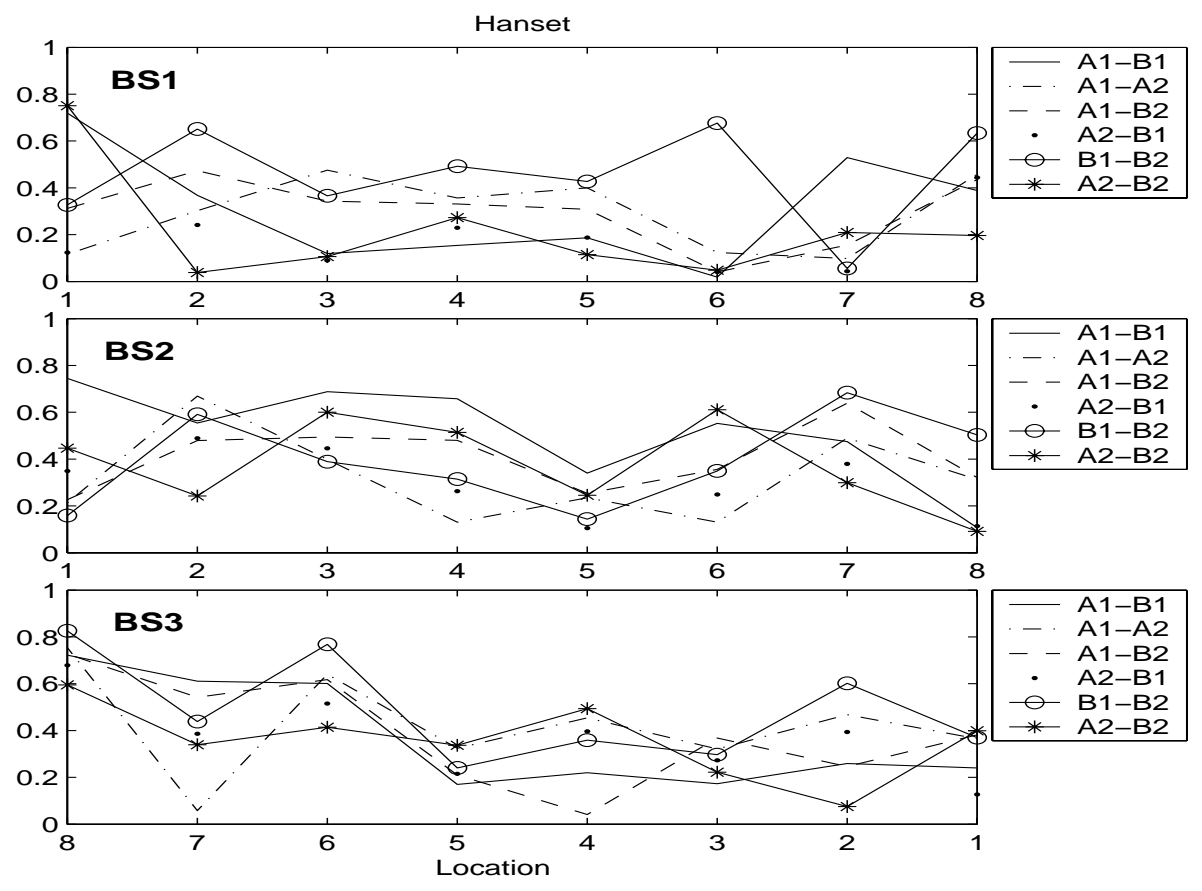

a. Handset
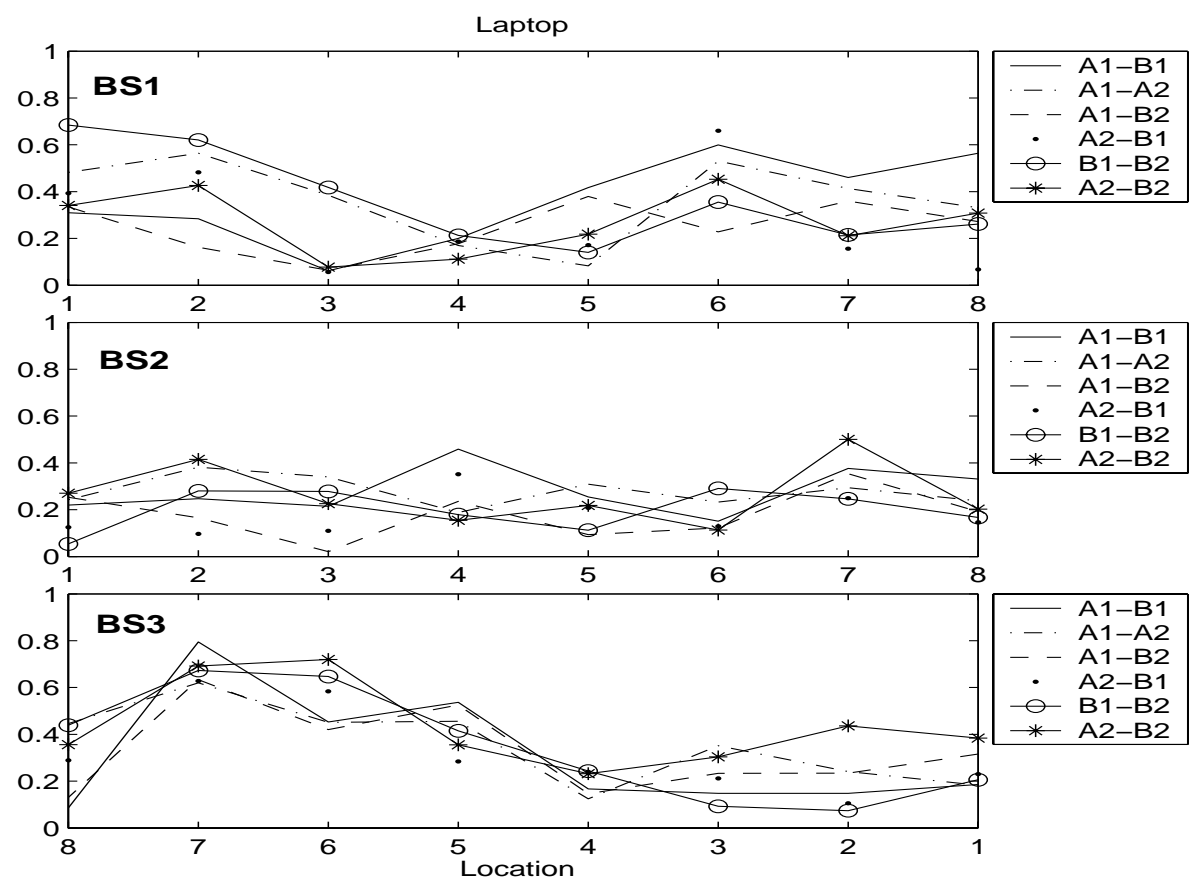

b. Laptop

Figure 6. Spatial correlation at the handset and the laptop along the corridor for indoor-indoor and outdoor-indoor setup

is observed that the highest value of the spatial correlation coefficient is 0.85 and the lowest value is 0.05 . The analytical correlation coefficient obtained from a widely used Jake model $J_{o}\left(\frac{2 \pi d}{\lambda}\right)$, are $0.47,0.30$ and 0.26 for a antennas separated distance of $0.25 \lambda, 0.5 \lambda$ and $0.75 \lambda$ respectively. In general, the measured spatial correlation coefficient between receiving antenna elements hardly reflects the 
separation between them. Mutual coupling, differences in the antenna radiation patterns which can be expected from real life scenario must be the main reason for the disagreement in the measured and theoretical results.

\subsection{Channel Capacity}

As mentioned in the previous chapter it is possible to transmit up to $\min \left(N_{t}, N_{r}\right)$ subchannels in a MIMO system consisting of $N_{t}$ transmitting antenna and $N_{r}$ receiving antennas. Therefore there is a significant increase in the channel spectral efficiency or the channel capacity. The theoretical amount of information or capacity the MIMO system can convey is defined as (Telatar, 1999).

$$
C=\log _{2}\left(\operatorname{det}\left(I+\frac{S N R}{N_{t}} H * H^{H}\right)\right)=\sum_{k=1}^{K} \log _{2}\left(1+\frac{S N R}{N_{t}} \lambda_{k}\right)
$$

where ()$^{H}$ denotes the Hermitian transpose operator; $K=\min \left(N_{t}, N r\right)$ and $\lambda_{k}$ is the $k^{t h}$ non zero eigenvalue derived from the eigenvalue decomposition of the normalized matrix $H$. This formula is applied to the case where the channel state information (CSI) is only known at the receive side, and therefore the most reasonable way of distributing the transmitted power is to distribute it equally to each transmitting antennas.

It has been demonstrated in (Andersen1, 2000) that for a MIMO system with wide angular spread at both ends a diversity order of $N_{t} N_{r}$ can be obtained. Basically, by only knowing the CSI at the receive side it is possible to obtain the diversity gain at the receive side and full diversity order. But the mean gain is degraded because the transmitted signal is not matched with the channel. However, in order to have full gain, knowledge of the channel must be available at both the transmitter and receiver. It has been shown that when the CSI is known at both ends of the transmission link, and with the total transmitted power constraint the maximum channel capacity can be achieved by using the waterfilling algorithm. Assuming that the noise variances are the same for all receive branches and the noise at each branches are mutually uncorrelated, the MIMO channel capacity using the waterfilling method can be described by

$$
C=\sum_{k=1}^{K} \log _{2}\left(1+\lambda_{k} \gamma_{k}\right)
$$

where $\gamma_{k}=\max \left(\mu-\frac{1}{\lambda_{k}}, 0\right)$ and the water level $\mu$ is chosen such that $\sum_{k=1}^{K} \gamma_{k}=S N R$.

When the number of the transmitting antennas is greater than the number of the receiving antennas, the advantage of having the CSI at the transmit side becomes more significant. This is because the transmitted power can be focused into the channels which have non zero eigenvalues. Meanwhile for the case where the CSI is only available at the receive side, the transmitted power is equally distributed over all channels, including the channel with zero eigenvalues. This leads to a loss in the received power and as a result reduction in the capacity.

The combination of the normalization process and the use of the fixed reference SNR imply that at the receiver we have a perfect power amplifier and attenuator so that the average SNR is always kept constant. Therefore the effect of path loss caused by the separation between the transmitter and receiver and the measured environment is always circumvented.

It should be noted that there is distinct difference in the reference SNR and the signal to noise ratio in the measurement $\mathrm{SNR}_{m}$. In our measurement the system noise is mainly due to the quantization noise and the correlation noise. Even though the system noise somehow correlated with the input power, in most of the measured locations the measured $\mathrm{SNR}_{m}$ is guaranteed to be well more than 40dB. Depending of its relative power as compared with the input signal power, the noise could make the channel matrix become more uncorrelated as it actually is. Therefore, we want to have a system with high $\mathrm{SNR}_{m}$ so that the effect of noise is limited. Low value of the $\mathrm{SNR}_{m}$ could introduce erroneous eigenvalues and therefore erroneously high value of the channel capacity especially with high reference SNR (see (Gans, 2002)). Aware of this problem we use a reference SNR value of $20 \mathrm{~dB}$. 
In the following, we will consider both scenarios where the CSI is assumed to be known perfectly only at the receiver and at both ends on the transmission link. For each placement of the BS, the measured data collected from all measured locations of the handset and laptop were used to calculate the distribution of the channel capacity. This will give an overview picture on the effect of the measurement scenario e.g. indoor-indoor, outdoor-indoor and the laptop/handset on the theoretical capacity (figure 7).

From this figure, it is observed that the indoor-indoor scenarios with the handset as a receiver shows the largest capacity. The median capacity is accountable for at least $80 \%$ of the one obtained from the channel matrix with complex Gaussian IID entries. The availability of CSI at the transmit side leads to a significant improvement in the achievable capacity. An increase of $20 \%$ in the median capacity as compared to the case where the CSI only known at the receiver is observed. To illustrate the advantage

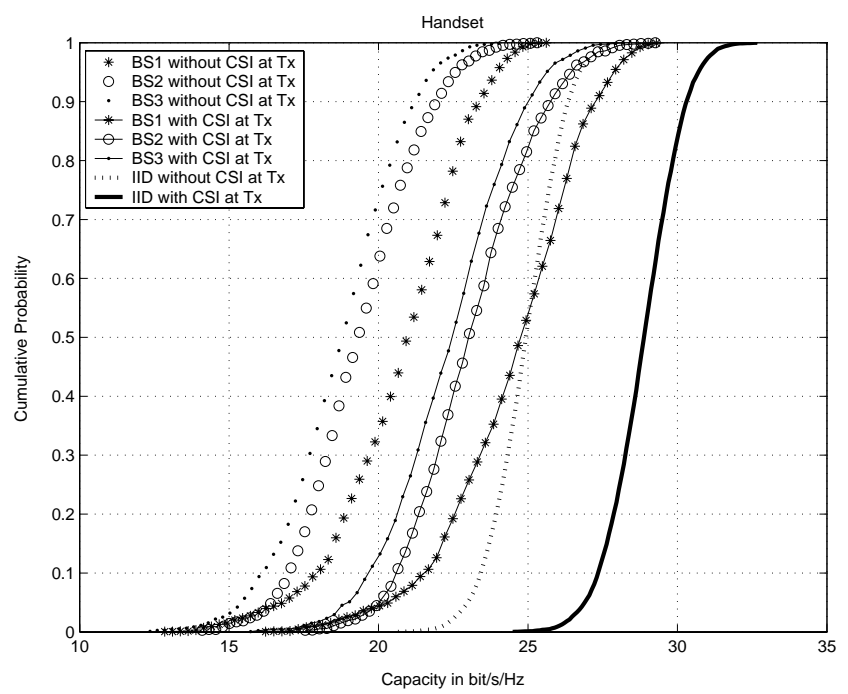

a. Handset

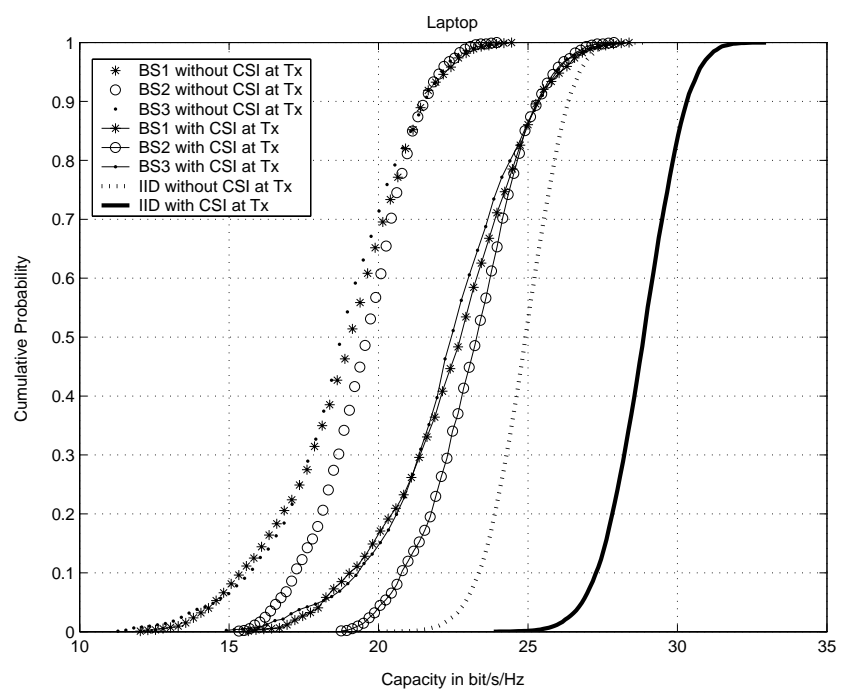

b. Laptop

Figure \%. Distribution of the channel capacity with the handset and laptop as receiver obtained from all measured sites at the with the system $S N R=20 \mathrm{~dB}$

of having the channel knowledge at the transmitter as a function of the reference SNR we calculated the improvement or gain in the channel capacity where the CSI is known at both sides as compared to the case it is only known at the receiver figure (8). At lower SNR level there is a considerable increase in the capacity of up to $240 \%$. The advantage decreases gradually as the reference SNR value increases. 
It is interesting to see that the capacity gain in the IID case is always lower than the one obtained from the measured data. The reason behind it is that the waterfilling method tries to distributed more power into the channels with high gains or high eigenvalues. Together with the fact that the eigenvalues achieved from the measurement distribute over a larger range than those in the IID case, the largest eigenvalue also has a higher value. Therefore the effect of using the waterfilling method becomes more prominent in the measured data than in the IID case.
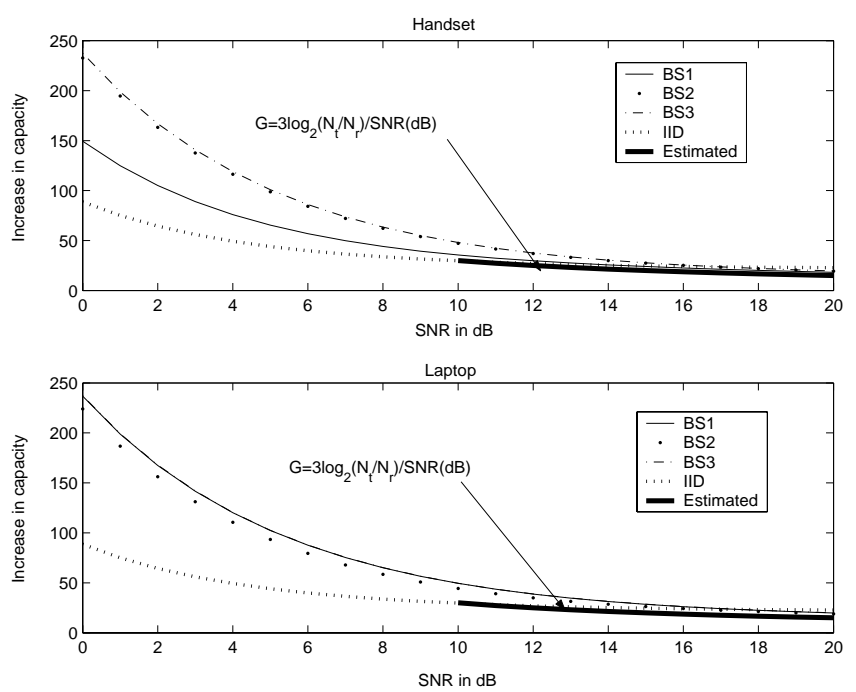

Figure 8. The advantage of having the knowledge of the channel at the transmitter as a function of the SNR, in percentage

Without going into the actual numerical calculations of the channel capacity the advantage of having the CSI at the transmitter can be estimated for the IID case when the number of transmitting antennas $N_{t}$ is much larger than the number of receiving antennas $N_{r}$. The approximation of the MIMO channel capacity for this case can be found in (Andersen2, 2000). When the CSI is not known at the transmit side the channel capacity can be approximated as

$$
C=N_{r} \log _{2}(1+S N R)
$$

and when the CSI is known at the transmitter

$$
C=N_{r} \log _{2}\left(1+S N R \frac{N_{t}}{N_{r}}\right)
$$

It can be seen that under such circumstances the capacity of the MIMO channel increases linearly with the number of receiving antennas regardless of whether the channel is known at the transmitter or not. It follows that the advantage of knowing the CSI at the transmit side or the gain in the channel capacity for $N_{t} \gg N_{r}$ can be estimated as

$$
G=\frac{N_{r} \log _{2}\left(1+S N R \frac{N_{t}}{N_{r}}\right)}{N_{r} \log _{2}(1+S N R)}-1=\frac{\log _{2}\left(1+S N R \frac{N_{t}}{N_{r}}\right)}{\log _{2}(1+S N R)}-1 \approx \frac{3 \log _{2} \frac{N_{t}}{N_{r}}}{S N R(d B)}
$$

The approximation sign is for the case the value of SNR is much greater than 1 . In the same figure 8 we plot the approximation of the capacity gain for the SNR values ranging from $10 \mathrm{~dB}$ to $20 \mathrm{~dB}$ with the $8 \times 4$ MIMO setup. The result matches with the numerical calculation for the IID data and the measured data quite well.

To highlight the site dependency of the multipath richness of the measured MIMO radio channel, we calculate the mean capacity at each measured location. The mean values of the capacity corresponding to the measured locations, the BS's placement and the receiver's types laptop/handset are plotted in figure 9.a and 9.b.

It is observed that at the same measured location the mean channel capacity does not change significantly by switching the receiver from the handset to the laptop. In some cases, having the handset 

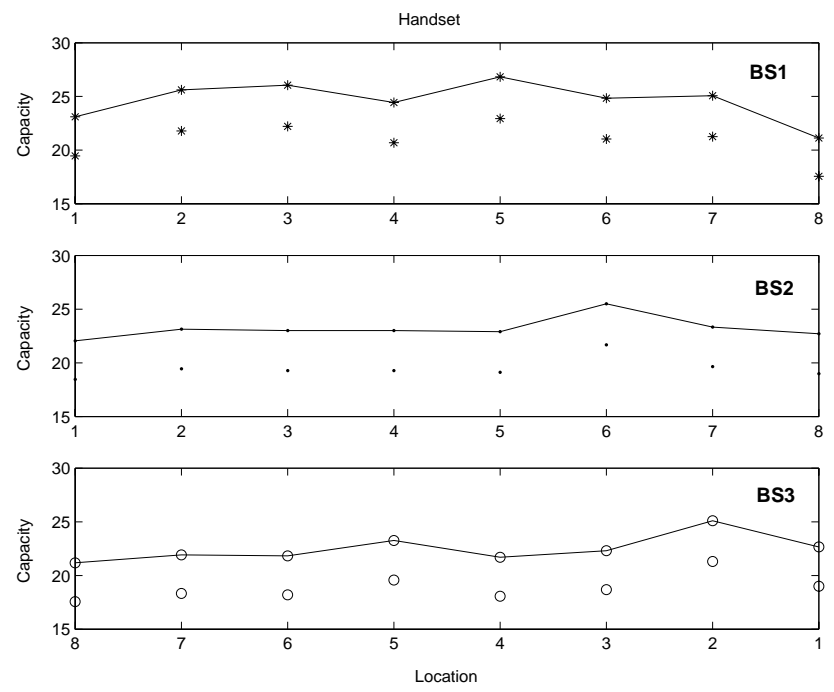

a. Handset
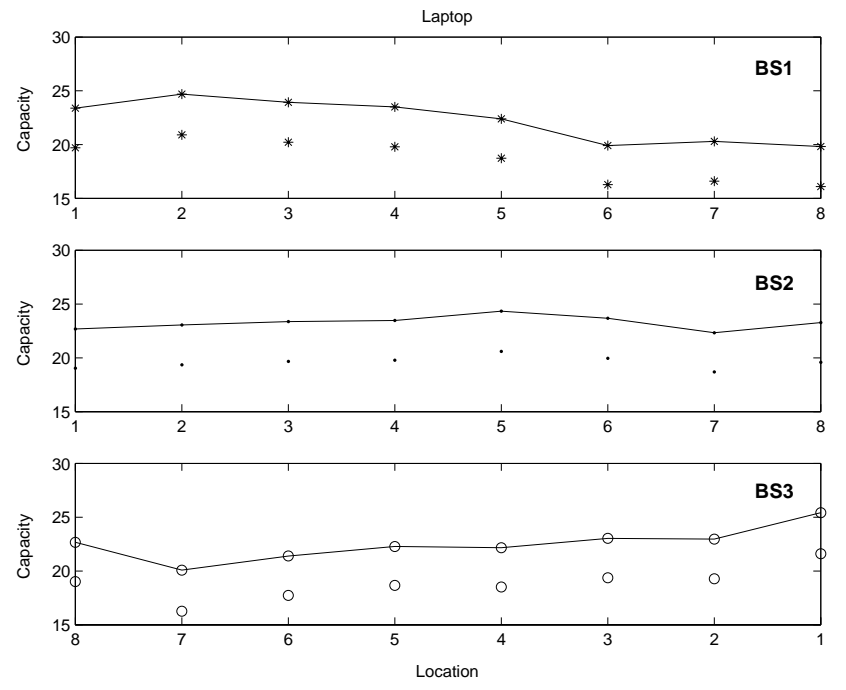

b. Laptop

Figure 9. The dependency of the mean channel capacity with the handset and laptop as the receiver along the corridor for indoor-indoor and outdoor-indoor setup, $S N R=20 \mathrm{~dB}$, symbol with solid line: Channel is known at Tx, symbol only: Channel is unknown at Tx. Order of the receiver's location has been reversed in the third row

as the receiver other than the laptop could lead to a higher mean channel capacity (i.e. location 5 BS1, location 6 BS2 and location 2 BS3). This can be explained by the fact that the handset and laptop's antennas are oriented on perpendicular directions. The main beams of the antenna elements at the laptop are faced toward the open door, the place where the signals with high energy are expected to arrive. Therefore the laptop has a higher chance of capturing correlated multipath components. Other thing could be contributed is that the antenna in the laptop has narrower beamwidth (more directional), which can be the result of larger ground plane, than the handset's (almost omnidirectional). Narrower radiation pattern will result in fewer multipath components being received and an increment in the correlation of the received signals at the receiving antennas. Consequently less uncorrelated multipath components impinging on the receiving antennas are captured, even though the distance between antenna elements at the laptop is slightly larger. This will give rise to the reduction of the channel capacity when the laptop is the receiver.

As also investigated in (Kyritsi1, 2002) and (Kyritsi2, 2002) for measured MIMO radio channel along the corridor, it is expected that those locations with high path loss or equivalently lower received power also have a poor multipath scattering characteristics. The corridor forming a wave guide will 
limit the angular spread and the propagation is dominated by a deterministic effect. As a result only components which have enough energy are able to arrive at the receiving antennas. The lack of multiple uncorrelated paths with approximately equal energy will reduce the multipath richness of the environment experienced by the receiving antennas. However, a modest decrement of the capacity along the measured location is only observed in the indoor-indoor scenario with the laptop as a receiver. Roughly speaking the channel multipath richness is independent of the measured locations as well as the type of receiver (either the handset or the laptop).

\section{Influence of large-scale variation to the channel capacity}

The multipath richness of the radio channel or more specifically the amount of information which can be conveyed is important for any MIMO system. However, for any wireless system to work properly the fundamental requirement on energy per bit symbol over the noise level must be fulfilled. Therefore, in a thorough assessment of the performance of any MIMO system these two fundamental characteristics: the radio channel multipath richness and the amount of received power should not be separated. It should be mentioned that having both the multipath richness and the averaged received power information is one of the advantage of measuring the MIMO radio channel.

Combining both the received power and multipath richness in the assessment of MIMO performance have been mentioned in (Wallace, 2002), (Svantesson, 2003) and (Mcnamara, 2003). In (Wallace, 2002) and (Svantesson, 2003) the measured channel matrices is normalized by a normalization factor which is the average power of the channel coefficients calculated from the entire measured data set. The channel capacity was then calculated using the normalized channel matrixes and a reference SNR. In (Mcnamara, 2003), the capacity is calculated by directly using the measured channel matrix and very high reference SNR such that after compensating for the path loss we still have positive received power in $\mathrm{dB}$ for all measured data set. However, the reference SNR should be chosen with care as the overall SNR (i.e. after compensating for the power loss) cannot be much larger than the actual $\mathrm{SNR}_{m}$ at the receiver.

Herein we use another approach where the reference SNR is not used, instead the SNR calculated as the ratio of the mean received power and a reference noise level, is used directly in the calculation of the MIMO ergodic capacity, that is

$$
\overline{S N R} \approx \frac{\overline{P_{\text {received }}}}{\sigma_{\text {noise }}^{2}}
$$

Note that we neglect the noise power which is inherently contained in the mean received power. This explains the $\approx$ sign in equation $(10)$.

The capacity of the MIMO channel using this approach can be described by

$$
C=\sum_{k=1}^{K} \log _{2}\left(1+\frac{\overline{S N R}}{N_{t}} \lambda_{k}\right)
$$

And when the CSI in known at both link ends and the waterfilling is used

$$
C=\sum_{k=1}^{K} \log _{2}\left(1+\lambda_{k} \gamma_{k}\right)
$$

where $\gamma_{k}=\max \left(\mu-\frac{1}{\lambda_{k}}, 0\right)$ and the water level $\mu$ is chosen such that $\sum_{k=1}^{K} \gamma_{k}=\overline{S N R}$.

In the equations above we use the mean received power for one SISO link as the total transmitted power for the MIMO system under consideration. Thereby the significant increase in the channel capacity of the MIMO system over the SISO system is highlighted.

As illustrated in (Amitay, 2001) the influence of the high measurement noise level or low measurement signal to noise ratio $\mathrm{SNR}_{m}$ to the estimated channel capacity becomes significant when: 
i) The channel is correlated or in an extreme case a reflectiveness far field free space wave propagation.

ii) Using rather high reference SNR value as compared to the $\mathrm{SNR}_{m}$ thereby the effect of erroneous eigevalues to the channel capacity is exaggerated.

In order to calculate the $\overline{S N R}$ stated in equation (10) care must be taken in choosing the noise level so that with this $\overline{S N R}$ the channel capacity value can be estimated with high reliability. At the same time, the variation of the mean received power is also reflected in the channel capacity. Taking these requirements into consideration and with the note that our measurement MIMO channel is partly correlated and it is measured with high $\mathrm{SNR}_{m}$, we decided to chose a noise level of $-95 \mathrm{dBW}$ for all receive branches and all measured data. As a result, the average SNR value as calculated in equation (10) ranges from $-3 \mathrm{~dB}$ to $40 \mathrm{~dB}$. Using this noise level, we calculate the capacity of the MIMO system for two cases where the CSI is assumed to be known at both link ends and at the receiver only. The distribution of the capacity of all measured locations for the indoor to indoor measurement and outdoor to indoor measurement are illustrated in figure 10.
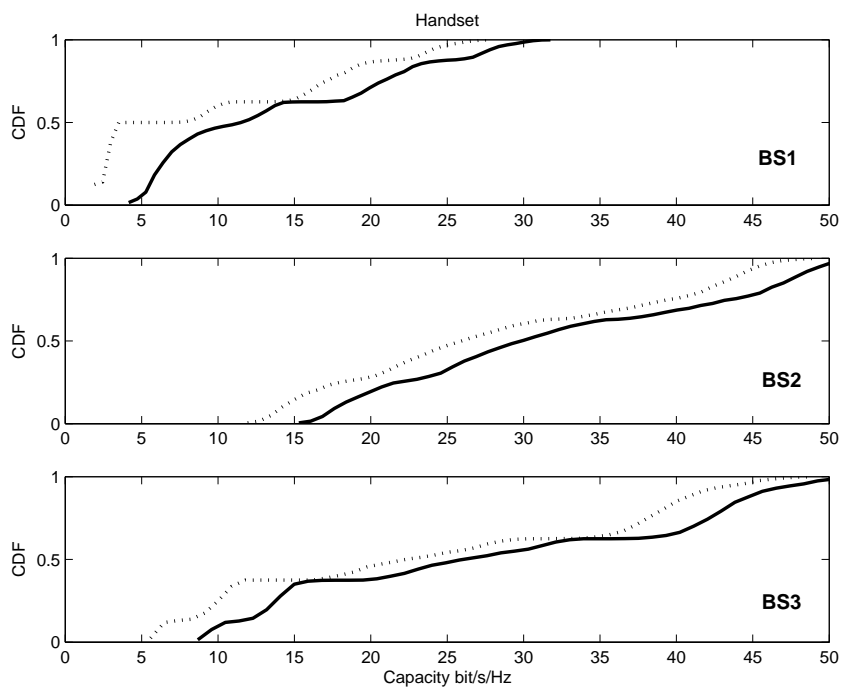

a. Handset
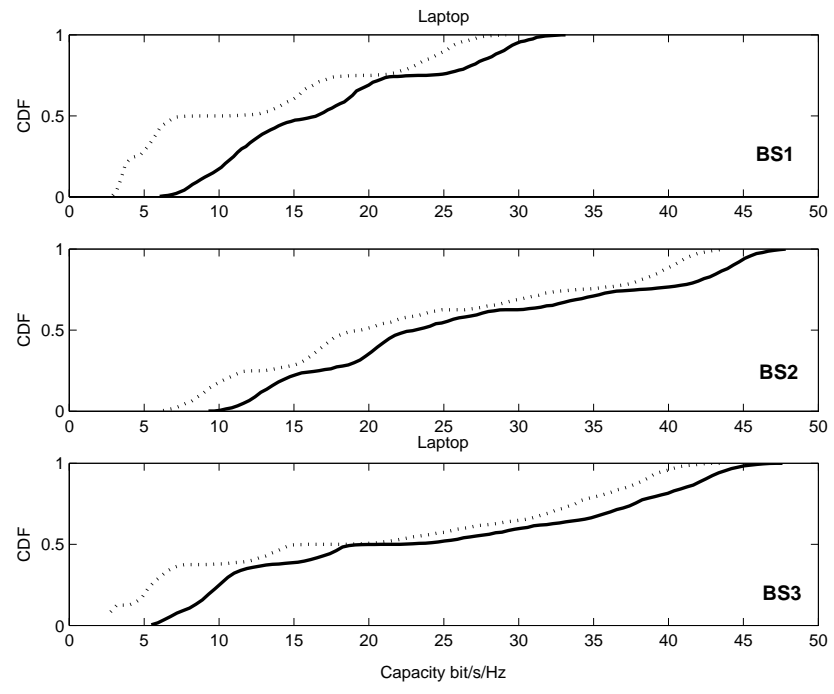

b. Laptop

Figure 10. Distribution of the capacity of the handset and laptop, using the average SNR for three measurement scenario, Dot lines: Channel is unknown at Tx, Solid lines: Channel is known at Tx 
In general, we are interested in the relative change of the MIMO system capacity from one receiver's location to another where both the mean received power and the environmental multipath richness are included. The results of the site dependency of the MIMO capacity calculated in this way are illustrated in figure 11. Note that the mean capacity at one measured location is obtained by averaging the ergodic capacities at all measured snapshots. Because the mean received power at one measured location is retained, there is a larger spread in the MIMO capacity along the corridor.

By comparing figure 9 and figure 11 significant difference in the trend of the capacity along the measurement location can be noted. On one hand, when there are perfect amplifier and attenuator at the receiver so that the SNR for one SISO link is unchanged regardless of the receiver's location, the capacity or the multipath richness of the measured environment along the corridor almost remains the same. On the other hand, when the capability of the amplifier and/or attenuator at the receiver is limited, which is common in practice, the variation in the mean received power has greater influence on the change of the channel capacity than variation in the multipath richness properties of the MIMO radio channel does.
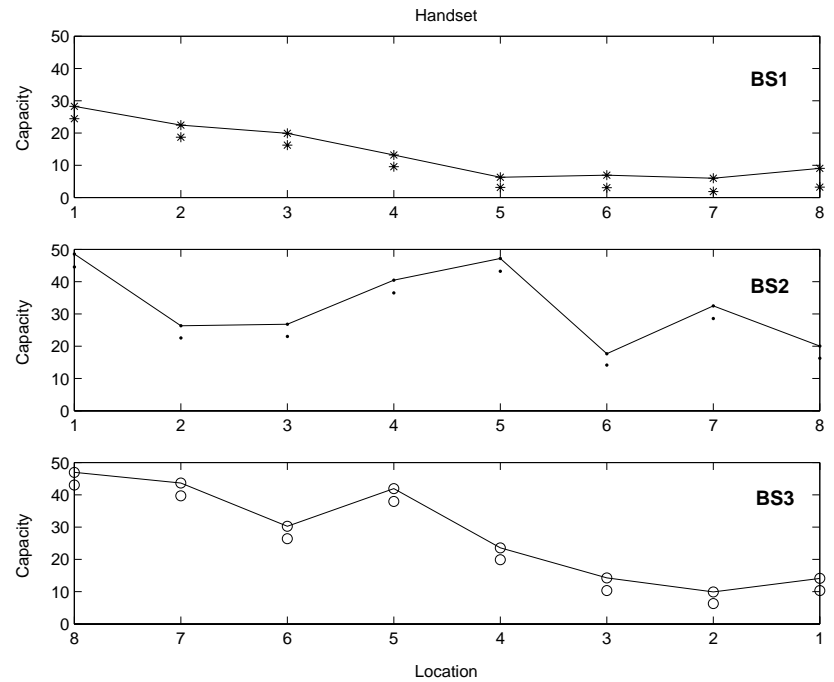

a. Handset
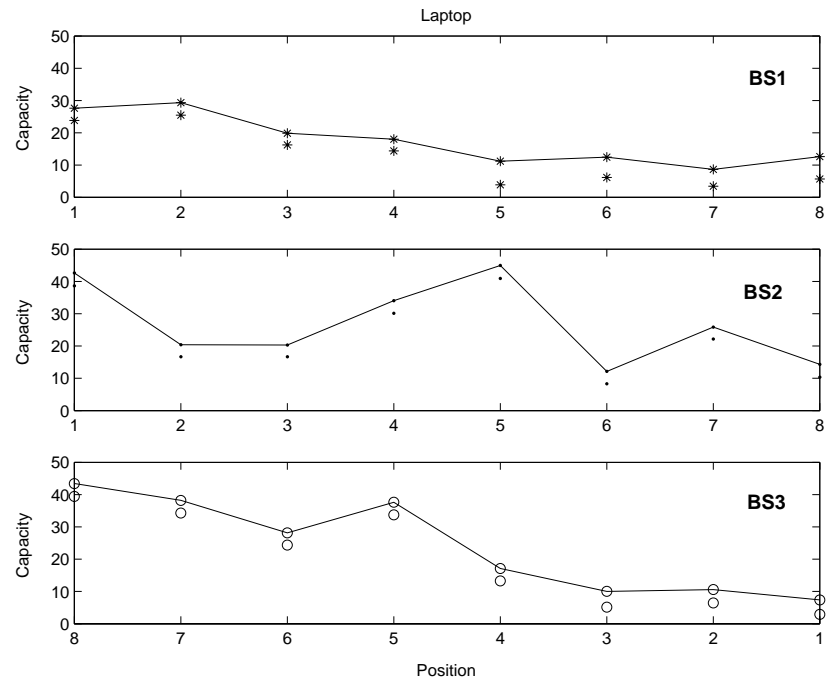

b. Laptop

Figure 11. The mean channel capacity with the handset and laptop as the receiver along the corridor, using the average SNR, symbol with solid line: Channel is known at Tx, symbol only: Channel is unknown at Tx 
The advantage of having the CSI at the transmitter over the case it is unknown in terms of the gain in the mean capacity is also illustrated. As expected the ergodic capacity when the channel is known to the transmitter is always higher than the ergodic capacity when the channel is unknown. However, the general trend is that the gain decreases as the received power increases. This indicates the fact that the advantage of knowing the channel at the transmitter reduces at higher SNR values.

\section{Conclusion}

In this paper, using a narrowband analysis we have evaluated the performance of the $8 \mathrm{x} 4$ measured MIMO system for indoor-indoor and outdoor-indoor scenarios. To mimic a real MIMO system, a prototype of a BS with eight outputs and prototypes of small terminals such as mobile handset and laptop with four patch antennas were deployed in the measurement. General properties of the measured MIMO radio channel as well as its distinct characteristics those adhere to the measurement environment and scenarios have been analyzed and discussed. The mean received power decreases steadily as the distance between the transmitting antennas and the receiving antennas increases. The multipath richness characteristics of the measured radio channel is almost independent of the measured locations as well as the type of the receiver (either handset or laptop). The distributions of the eigenvalues or the gains of the subchannels observed from the measurement data were spread over a larger range than that of the theoretical channels with identical independently distributed (IID) complex Rayleigh fading components. It is shown that when there are perfect amplifier and/or attenuator so that the mean SNR remains constant i.e. $20 \mathrm{~dB}$, the median capacity of the measured MIMO channel is accountable for at least $80 \%$ that of system with IID components. This indicates that in order to obtain full diversity gain and diversity order it is still make sense to deploy multiple antennas in a small terminal. The gain in channel capacity which is resulted from knowing the channel at the transmitter is significant for low SNR. At the SNR value of $20 \mathrm{~dB}$, using the measured data we show that by knowing the channel at both ends of the transmission link it is possible to obtain up to $20 \%$ increase in the channel capacity as compared with the case where the channel is known at the receive side only. However, the gain gradually reduces at higher received power or higher SNR value. We estimate the channel capacity where both the change in the mean received power and the change in the MIMO radio channel multipath characteristics are included. The analysis show that the variation in the mean received power can have greater influence on the overall system performance than the change in the multipath scattering property.

\section{Acknowledgement}

Nokia is kindly acknowledged for their financial contribution in the measurement campaign. The authors would like to thank Wim A.T. Kotterman, Jesper Ødum Nielsen and Kim Olesen for carrying out the measurements and data post-processing and the fruitful discussions are also gratefully acknowledged.

\section{References}

M.Steinbauer, A.F.Molisch, and E.Bonek The double-directional radio channel. IEEE Antennas and Propagation Magazine, vol. 43, pages 51-63, August 2001.

R.Thoma, D.Hampicke, M.Landmann, G.Sommerkorn, and A.Richter MIMO measurement for double-directional channel modelling. IEE Seminar on MIMO: Communications Systems from Concept to Implementations, pages 1-7, December 2001.

J.Medbo and J.E.Berg Spatio-Temporal channel characteristics at $5 \mathrm{GHz}$ in a typical office environment. IEEE Vehicular Technology Conference vol. 3, pages 1256-1260, October 2001.

T.Fugen, G.Sommerkorn, J.Maurer, D.Hampicke, W.Wiesbeck and R.Thoma. MIMO capacities for different antenna arrangements based on double directional wide-band channel measurements. The 13th IEEE International Symposium on Personal, Indoor and Mobile Radio Communications vol.4, pages:1777 - 1781, September 2002 
D.Chizhik, J.Ling, P.W.Wolniansky, R.A.Valenzuela, N.Costa, and K.Huber Multiple-input-multiple-output measurements and modeling in Manhattan. IEEE Journal on Selected Areas in Communications vol. 21, pages 321-331, April 2003.

D.P.Mcnamara, M.A.Beach, P.Karlsson, P.N.Fletcher. Initial characterisation of multiple-input multiple-output (MIMO) channels for space-time communication. IEEE VTS-Fall VTC 52nd, pages 1193 -1197, Semtember 2002.

J. Kivinen, P. Suvikunnas, L. Vuokko, and P. Vainikainen Experimental investigations of MIMO propagation channels. IEEE Antennas and Propagation Society International Symposium, vol. 3, pages 206-209, June 2002.

J.P.Kermoal, P.E.Mogensen, S.H.Jensen, J.B.Andersen, F.Frederiksen,T.B.Sorensen, K.I.Pedersen Experimental investigation of multipath richness for multi-element transmit and receive antenna arrays. IEEE 51st VTC 2000, vol. 3, pages 2004-2008, 2000.

K.Yu, M.Bengtsson, B.Ottersten, P.Karlsson, D.McNamara and M.Beach Measurement Analysis of NLOS Indoor MIMO Channels. IST Mobile Communications Summit, 2001, pages $277-282$.

T.Svantesson, J.Wallace On signal strength and multipath richness in multi-input multi-output systems. IEEE International Conference on Communications, vol.4, pages 2683 - 2687, May 2003.

M.B.Knudsen, G.F.Pedersen Spherical outdoor to indoor power spectrum model at the mobile terminal. IEEE JSAC Issue: 6 vol.20 pages 277 - 282, August 2002.

I.E.Telatar Capacity of Multi-antenna Gaussian Channels. European Transactions on Telecommunications, No.6, vol. 10, pages 585-595, 1999.

J.B.Andersen Antenna arrays in mobile communications: gain, diversity, and channel capacity. IEEE Antennas and Propagation Magazine, Issue: 2, vol.42, pages 12-16, 2000.

M.J.Gans, N.Amitay, Y.S.Yeh, Hao Xu, T.C.Damen, R.A.Valenzuela, T.Sizer, R.Storz, D.Taylor, W.M.MacDonald, Cuong Tran and A.Adamiecki Outdoor BLAST measurement system at $2.44 \mathrm{GHz}$ : calibration and initial results. IEEE Journal on Selected Areas in Communications, Issue: 3, vol.20, April 2002.

J.B.Andersen Array gain and capacity for known random channels with multiple element arrays at both ends. IEEE Journal on Selected Areas in Communications, Issue: 11, vol.18, pages 2172 - 2178, November 2000.

P.Kyritsi, N.Kadri, E.Thang, D.C.Cox Signal correlation in a hallway environment using waveguide mode analysis. IEEE VTC, 2002, vol.2, pages $787-791,2002$

J.W.Wallace, M.A.Jensen, A.L.Swindlehurst, B.D.Jeffs Experimental characterization of the MIMO wireless channel: data acquisition and analysis. IEEE TWC, Issue: 2, vol.2, pages 335 - 343, March 2003.

T.Svantesson, J.Wallace, On signal strength and multipath richness in multi-input multi-output systems IEEE International Conference on Communications vol.4, 2683 - 2687, 2003

P.Kyritsi, D.C.Cox, R.A.Valenzuela, P.W.Wolniansky Effect of antenna polarization on the capacity of a multiple element system in an indoor environment. IEEE Journal on Selected Areas in Communications, Issue: 6, vol.20, pages 1227 - 1239, August 2002.

D.P.Mcnamara Characterisation and Investigation of Multiple-Input Multiple-Output Wireless Communications Channel. PhD thesis University of Bristol Faculty of Engineering, 2003.

N.Amitay, M.J.Gans, H.Xu, R.A.Valenzuela Effects of thermal noise on accuracy of measured BLAST capacities. IEE Electronics Letter, Issue: 9, vol.37, pages 591 - 592, 2001 
indoor_journal.tex; 19/04/2005; 0:13; p.18 